Musées, Patrimoine et Culture scientifiques et techniques

$140 \mid 2012$

mars-avril 2012

\title{
De la diffusion des sciences à l'Agenda 21
}

From Science diffusion to the Agenda 21

\section{Agathe Pellas et Marie-Christine Hergault}

URL : http://journals.openedition.org/ocim/1030

DOI : $10.4000 /$ ocim. 1030

ISSN : 2108-646X

Éditeur

OCIM

Édition imprimée

Pagination : 5-9

ISSN : 0994-1908

\section{Référence électronique}

Agathe Pellas et Marie-Christine Hergault, « De la diffusion des sciences à l'Agenda 21 », La Lettre de I'OCIM [En ligne], 140 | 2012, mis en ligne le 01 mars 2014, consulté le 01 mai 2019. URL : http:// journals.openedition.org/ocim/1030 ; DOI : 10.4000/ocim.1030 
Ce sont des sujets d'actualité, vivants, qui posent la question de la vérité scientifique, suscitant parfois polémiques et débats. À cela s'ajoute un traitement journalistique de la question environnementale qui a souvent tendance à renforcer le côté anxiogène. Se positionner en tant que référence scientifique sur des sujets où la science elle-même occupe parfois une position ambigüe est un exercice particulièrement délicat en raison de notre devoir d'objectivité et de transparence. Il n'est pas question de verser dans le catastrophisme, dans le militantisme ou à l'inverse de nier les risques que nous courrons. Le rôle d'un établissement comme Universcience est alors d'exposer l'état des connaissances et des incertitudes, d'expliquer les enjeux, de présenter les pistes à l'étude et les technologies qui feront la société de demain afin de permettre leur appropriation par le plus grand nombre. Il est aussi de travailler en étroite collaboration avec la recherche pour aider à l'émulation, à la diffusion de cette culture et même parfois être une plateforme de test en grandeur nature de certaines technologies.

En outre, ces sujets modifient la posture habituelle de culture scientifique. À cette diffusion de connaissances (fondamentaux, systèmes, mécanisme...), s'ajoute également la volonté d'inciter à l'action aussi bien nos publics que nous-mêmes.

Les études de public nous l'ont montré : le visiteur/ citoyen souhaite à la fois être rassuré et guidé dans son quotidien et il est pour ces sujets, à juste titre, particulièrement exigeant sur la cohérence entre le fond et la forme. Les extraits des enquêtes de publics ci-dessous en témoignent :

"Certains visiteurs attirent aussi l'attention sur le décalage qui peut exister entre le message que veut faire passer l'exposition et la pratique de l'établissement qui le diffuse : le gaspillage de papier généré par le jeu de l'empreinte écologique, le fait que l'exposition soit trop chauffée sont relevés comme n'étant pas en accord avec le message écologique... » (extrait de Ramos, E. L'exposition Changer d'Ére: des solutions pour un changement possible. Synthèse, DEP, CSI, CERLIS, 2007).

L'analyse des messages du livre d'or de cette exposition montre également que de nombreuses personnes s'interrogeaient suite à leur visite sur le mode de conception de l'exposition : est-elle éco-conçue? Quelle est son empreinte écologique ? Quels matériaux ont été utilisés ? Sont-ils recyclables ou recyclés?

Nous pouvons dire que l'expérience riche et variée acquise par Universcience depuis 2003 montre que les musées de sciences sont un bon lieu de communication et d'émulation pour une culture du développement

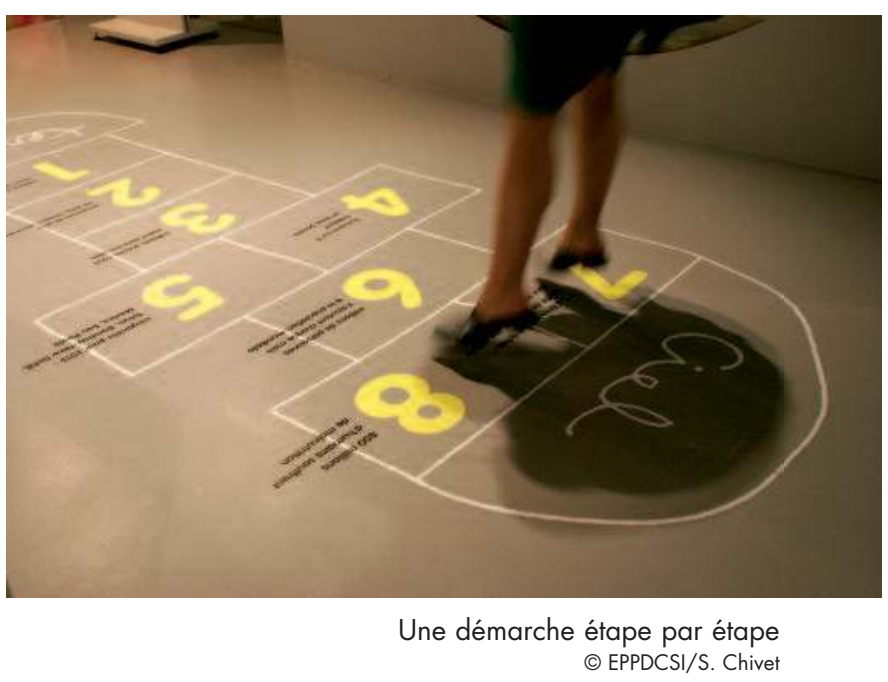

durable. C'est également une possibilité pour le musée d'élargir sa fréquentation à de nouveaux publics en s'adressant aux citoyens autant qu'aux « curieux de sciences $»$.

\section{Un message qui revisite nos pratiques}

Les musées de sciences ne se contentent plus d'être une interface entre les scientifiques et les publics, ils reconnaissent de plus en plus la nécessité de s'engager en donnant l'exemple et en s'appropriant eux-mêmes les solutions qui seront nécessaires à un développement durable de la société. Ainsi, un autre enjeu important pour l'établissement est de mettre en accord son fonctionnement avec le contenu de ses offres et des messages qu'il délivre.

La Cité des Sciences et de l'Industrie, par l'échelle de son bâtiment, la diversité de ses activités et sa mission elle-même, propose une configuration unique et particulièrement riche pour expérimenter la mise en place de process, de technologies et de management environnemental.

Dans un premier temps, la question de l'éco-responsabilité de l'établissement a été abordée en identifiant les grands projets à engager prioritairement sur des thématiques variées (énergie, achats, biodiversité, consommation de ressources, pollution des sols...). Il nous a paru indispensable de couvrir l'ensemble des activités de l'établissement : de l'activité administrative, à la gestion du bâtiment en passant, bien sûr, par la conception des expositions. Il s'agissait alors de mettre l'établissement en mouvement, de lancer les grands chantiers qui allaient nous permettre de nous approprier les enjeux principaux du développement 
durable et de monter en compétence sur des sujets à la fois ancestraux dans leur concept et nouveaux dans leur mise en application.

Dans ce cadre-là, nous avons commencé par revoir nos pratiques de gestion patrimoniale. Il a été nécessaire de conduire des études pour comprendre nos bâtiments, leurs différents usages, les contraintes qui pèsent sur leur exploitation et les objectifs qu'il nous faudra atteindre pour gérer plus durablement notre patrimoine bâti. Il a été question de réfléchir à une solution qui garantisse à la fois la pérennité des bâtiments, la sécurité et le confort des usagers ainsi qu'une meilleure gestion de nos ressources.

Cette réflexion globale nous a semblé importante pour construire un projet qui soit à la fois pertinent et soutenable pour l'établissement. Dans le contexte budgétaire contraint que l'on connaît, il est d'autant plus question de ne pas cloisonner les métiers, de conduire les projets strictement nécessaires en ayant suffisamment instruit le dossier pour avoir un objectif clair, concerté et répondant à l'intégralité des besoins. La mise en place se fait forcément par étapes dans un cadre comme celui de la Cité des Sciences où les dimensions (et donc les coûts) sont pharaoniques. Mais prendre le temps de mettre à plat toutes les problématiques du site a permis d'aboutir à un plan de travaux cohérent qui nous donne une feuille de route pour les années à venir. On pourrait peut-être comparer cela à la partition d'un chef d'orchestre qui est indispensable pour que tous les instruments jouent en harmonie sans fausse note. C'est bien entendu une vision un peu idéaliste mais cela permet déjà d'optimiser les travaux que l'on conduit, d'anticiper pour faire (toujours) un peu mieux.

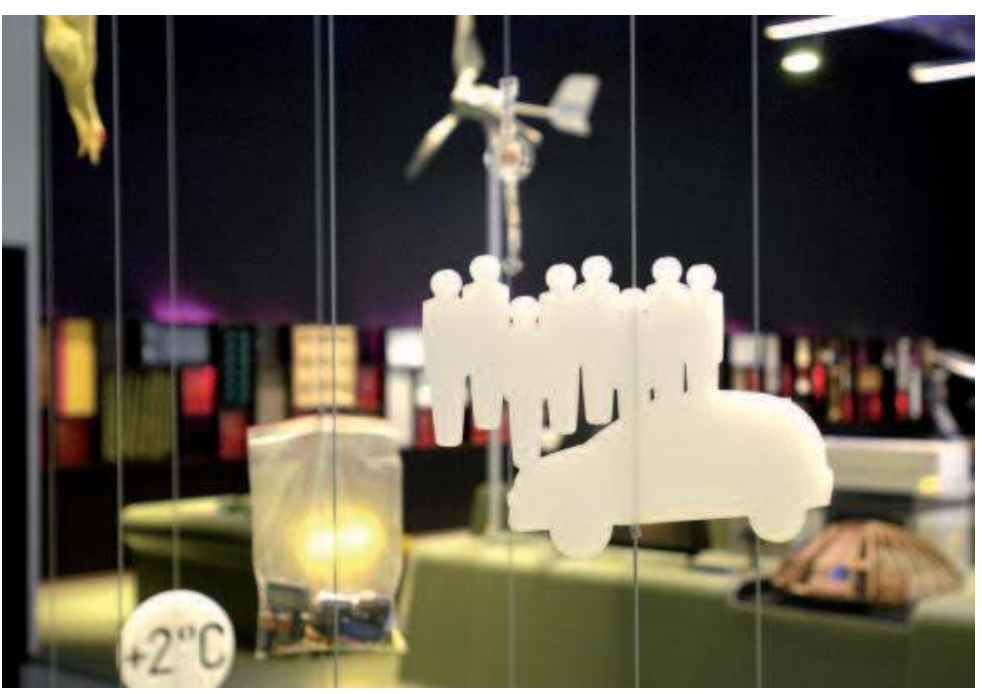

L'exposition Changer d'Ėre en 2007 (c) EPPDCSI/J.-P. Attal
Il a également fallu revoir nos principes de gestion administrative à l'aune du développement durable. Cela implique de repenser nos besoins, d'ajuster nos process, nos modes de travail. C'est une démarche longue, compliquée, où il n'y a pas de solutions toutes faites mais plutôt des raisonnements à intégrer dans chacune de nos tâches quotidiennes. C'est aussi et surtout un processus itératif sur des sujets non stabilisés, où on apprend en faisant, en avançant pas à pas vers plus d'équité et de durabilité. On peut citer notamment les passations de marchés où chaque renouvellement fait l'objet d'une expertise environnementale et sociale pour tenter de raisonner nos besoins et d'ajuster nos prescriptions et exigences aux enjeux environnementaux et sociaux. Pour la signalétique des expositions par exemple, nous avons fait un tour d'horizon de ce qui était disponible sur le marché et nous avons notamment pris le parti d'avoir recours à des bâches en textiles composites plutôt qu'en PVC classique, moins polluantes, en imposant leur recyclage dans le marché. L'année suivante nous avons appris que plutôt que de les recycler avec un coût énergétique et de transport important, nous pourrions plutôt les retransformer via un partenariat avec une société locale fabriquant des sacs et articles de maroquinerie dans le respect des gens et de l'environnement.

\section{Vers des expositions éco-conçues}

Un des grands chantiers encore en cours à Universcience est celui de l'éco-conception des expositions. C'est en 2006, à l'occasion de l'exposition Changer d'Ère que la Cité des Sciences et de l'Industrie a pour la première fois entrepris des actions, aussi modestes fussent-elles, sur cette problématique. En effet, l'exposition traitait des moyens de réduire son empreinte écologique au quotidien. Pour la crédibilité du propos, nous nous devions de construire une exposition en accord avec le message délivré.

Cette première expérience a été très enrichissante et a permis d'appréhender la complexité de la question et de poser les premières pierres de la démarche d'écoconception de nos expositions.

Suite à ce premier retour d'expérience, un guide à l'usage de nos concepteurs d'expositions a été rédigé. À travers Le guide d'éco-conception des expositions, notre intention était principalement de proposer une démarche, une philosophie de travail plutôt qu'un simple catalogue de produits et de références. En effet l'enjeu de l'éco-conception ne nous paraît pas être de choisir des matériaux écologiques mais de 


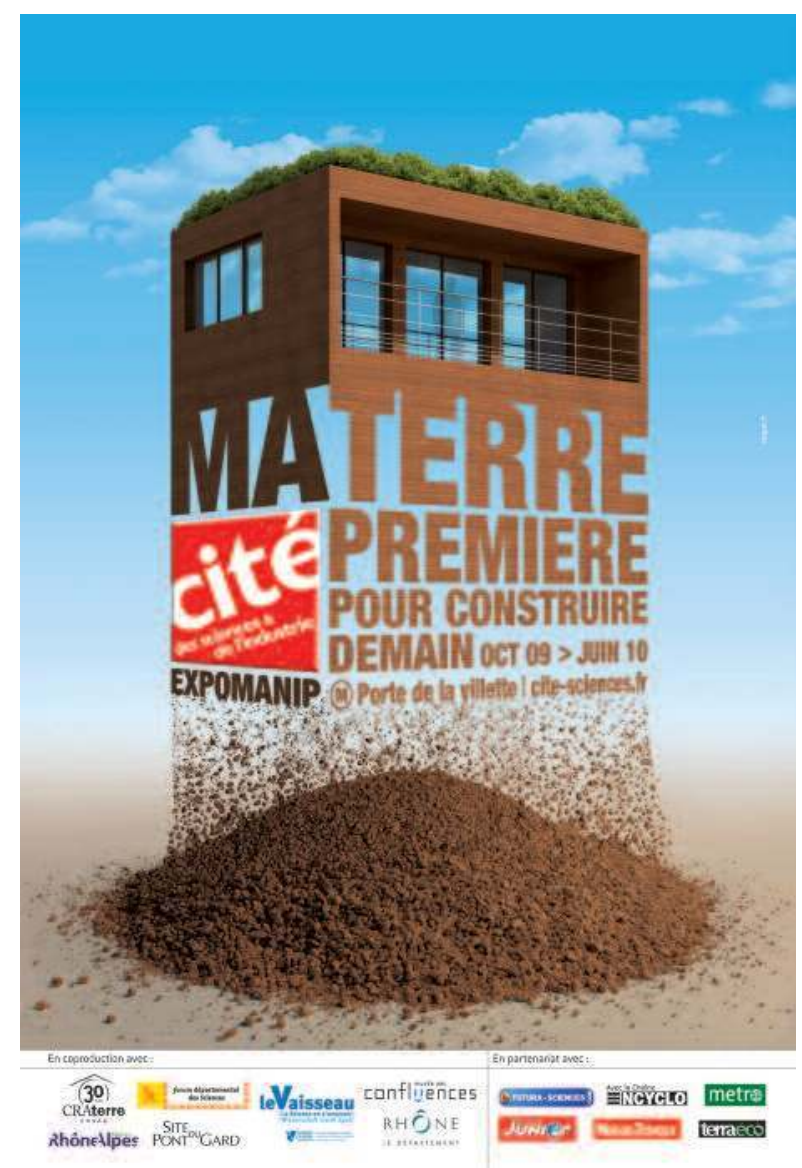

L'affiche de l'exposition Ma terre première en 2009 (c) EPPDCSI

revoir nos pratiques à travers le prisme du développement durable et ce, dès la phase de conception. C'est donc d'une approche globale dont il est question, qui vise à ce que les chefs de projet ou commissaires, mais aussi les différents intervenants, scénographes, designers, développeurs de présentations interactives, puissent y trouver des éléments de réflexion et des pistes de solutions relatives aux particularités de leur métier. Il est en effet essentiel que chacun des protagonistes se sente impliqué.

Ainsi, ce guide liste des préconisations phase par phase pour intégrer une démarche d'éco-conception dans la production d'une exposition. Il propose également une aide pour déterminer des objectifs à atteindre et évaluer le profil environnemental d'un projet. Pour cela, nous avons choisi de définir des cibles en nous inspirant des cibles HQE et en les adaptant au monde de la muséologie. Dans notre esprit, cet outil est appelé à évoluer et à être complété, notamment, nous l'espérons, par des retours d'expérience.

Lors de divers échanges ou colloques avec d'autres institutions muséales, nous avons perçu que ce guide, proposant à la fois une démarche détaillée, des outils d'analyse et de choix et des pistes de solutions et bien qu'initialement appliqué à notre processus interne de production, intéressait fortement d'autres institutions ou professionnels en France ou à l'étranger. C'est ainsi qu'aujourd'hui Le guide d'éco-conception des expositions est à la fois accessible aux collaborateurs internes et aux professionnels externes via le site d'Universcience.

Cependant il ne suffit pas que ce guide soit mis en ligne pour que son appropriation par les différents personnels concernés soit concluante. Les démarches d'éco-conception dépendent encore trop souvent de volontés individuelles et d'engagements personnels. Il est indispensable dinstitutionnaliser une culture commune qui soit à la fois portée par la hiérarchie et intégrée de plein gré par les utilisateurs aux méthodes de travail (chef de projet, concepteurs, scénographes...).

Pouvoir anticiper et imaginer son exposition avec les principes d'éco-conception doit devenir un savoirfaire intellectuel en relation avec tous les intervenants internes et externes. Afin d'aller dans ce sens, des formations internes, s'appuyant sur la démarche proposée dans le guide, complétées par une formation sur les matériaux ont été mises en place. Il est certain qu'il faudra encore quelque temps pour que la prise en compte des enjeux du développement durable aille de soi et ne soit pas vécue comme une contrainte supplémentaire.

L'exposition Ma Terre Première, exposition itinérante de $450 \mathrm{~m}^{2}$ présentée à la Cité des Sciences et de l'Industrie en 2009-2010, a été la première expérience d'utilisation du guide pour sa conception et sa production. Cette exposition est aussi la première exposition totalement éco-conçue par Universcience. Ce fut une expérience très riche en enseignements, qui nous a permis de recadrer les besoins et les priorités d'un projet et mieux se recentrer sur les fondamentaux du design d'exposition, qui se doit d'être à la fois fonctionnel, porteur de sens, esthétique tout en répondant aux enjeux environnementaux.

Les contraintes liées à l'éco-conception sont devenues une source de créativité et les réponses apportées en parfaite adéquation avec les contraintes liées à l'itinérance. Le temps d'étude a certes été un peu plus long, mais il a été compensé au cours des phases de réalisation et d'intégration qui se sont déroulées sans surprises ni plus-values. Il a fallu en outre consacrer du temps pour transmettre, communiquer les intentions et convaincre l'ensemble des intervenants qui ne gèrent pas forcément les mêmes priorités. 


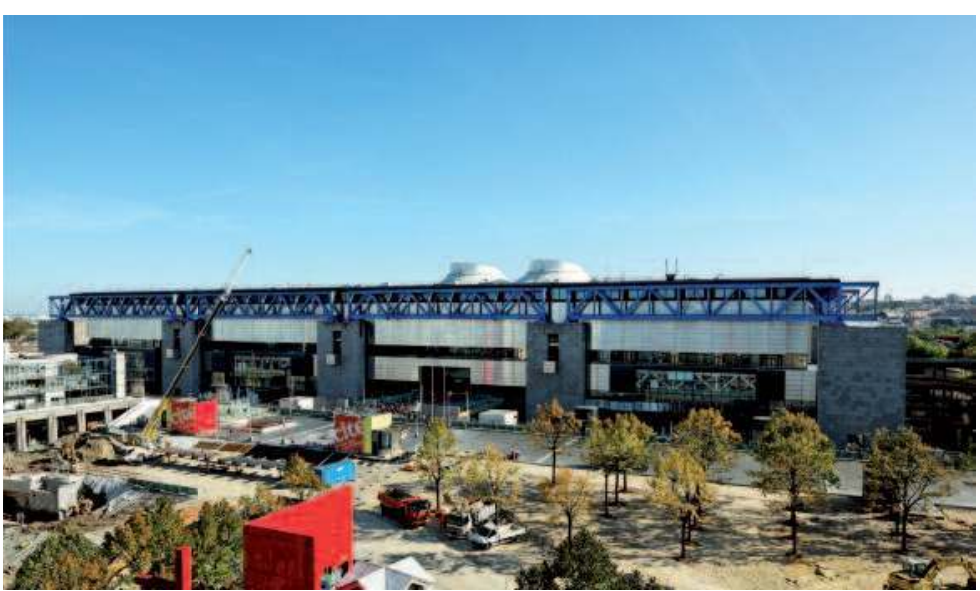

Le chantier de rénovation de l'accueil en 2011

() EPPDCSI

Les choix d'éco-conception n'ont pas engendré de surcoûts, le coût d'objectif a été respecté grâce à l'optimisation. Des économies sur les coûts de transport ont été réalisées grâce à la réduction du nombre de camions et sur la réduction du colisage. La définition d'objectifs à l'aide de cibles a permis de dresser un bilan environnemental et de communiquer les choix pour mutualiser cette expérience.

Une question nous a souvent été posée : cette exposition a-t-elle pu être éco-conçue en raison de sa thématique liée au développement durable ? Cela a effectivement été un moteur mais ce n'est pas une condition sine qua non. De nouvelles réponses inventives et spécifiques sont à trouver quels que soient les sujets abordés.
La formalisation d'une exposition par le biais d'une démarche éco-conçue, peut et se doit de devenir au même titre qu'un discours, un vecteur de médiation qui encourage les visiteurs à s'engager dans des pratiques plus respectueuses de notre environnement. Prendre le risque d'éco-concevoir c'est prendre le risque : de partager et de se battre, d'être modeste, d'oser déroger aux règles et aux habitudes, de prendre du temps, d'accepter de ne pas être parfait. Tout cela en fait, pourrait-on dire, juste un peu plus que d'habitude...

\section{Quels enjeux pour demain?}

Maintenant que nos premières armes ont été faites, l'enjeu pour Universcience est double : généraliser les pratiques et pérenniser la prise en compte du développement durable dans l'institution. Il y a des réticences à combattre mais c'est avant tout un projet d'entreprise, fédérateur, que nous nous efforçons de conduire en bonne intelligence avec tous les acteurs concernés. Pour insuffler, professionnaliser, suivre, et accompagner ce changement au niveau de l'établissement, nous avons toute une palette de possibilités dont la pertinence est à étudier : de la démarche autoproclamée à l'ISO 14001 en passant par l'Agenda 21. Quelle que soit la démarche adoptée, elle se nourrira des retours d'expérience et ne pourra se faire qu'avec l'adhésion de tous. 